\title{
The role of discourse relations in persuasive texts
}

\author{
Ines Rehbein \\ Leibniz ScienceCampus \\ IDS Mannheim / ICL Heidelberg \\ rehbein@cl.uni-heidelberg.de
}

\begin{abstract}
This paper investigates the use of explicitly signalled discourse relations in persuasive texts. We present a corpus study where we control for speaker and topic and show that the distribution of different discourse connectives varies considerably across different discourse settings. While this variation can be explained by genre differences, we also observe variation regarding the distribution of discourse relations across different settings. This variation cannot be explained by genre differences. We argue that the differences regarding the use of discourse relations reflects different strategies of persuasion and that these might be due to audience design.
\end{abstract}

\section{Introduction}

This contribution studies the use of discourse connectives in persuasive texts that have been produced in different communicative settings. Discourse connectives are highly ambiguous and polyfunctional and can vary across different dimensions, depending on the medium (spoken vs. written), the discourse situation (monologic vs. dialogic, formal vs. informal), the purpose of communication (informative vs. persuasive), and more. As we are most interested in investigating different strategies of persuasion, we focus on explicit markers of concessive and contrastive discourse relations, used by the speaker to provide a convincing argument that might persuade the hearer.

Work on discourse analysis and argumentation mining has highlighted the important role of discourse connectives for analysing argumentation structure (Felder, 2015; Stab and Gurevych, 2014; Eckle-Kohler et al., 2015). In addition, psycholinguistic studies have shown that explicit coherence marking not only improves sentence comprehension but also results in a more positive evalua- tion of the text by the reader (Kamalski et al., 2006). This suggests that discourse connectives might play an important role in persuasion strategies.

We take this hypothesis as our starting point and investigate how different dimensions of variation in persuasive texts can impact the linguistic behaviour of an individual speaker. To that end, we present a corpus study where we try to keep as many variables fixed and only vary the situational setting in which the texts were produced. We control for speaker, topic and function (i.e. persuasive texts) but vary the situational setting of text production.

The data we use in our analysis are political articles, interviews and talks produced by Noam Chomsky. Our data covers spoken and written texts and ranges from highly edited to less edited, including monologic as well as dialogic data.

The variation of discourse settings in our data is accompanied by changes regarding the audience in the different discourse situations. For interviews, the audience is rather small, often on a one-on-one basis, but the hearers have the means to interact with the speaker. This is different from the situation for public talks where we usually have a much larger audience that can be directly addressed by the speaker but has limited possibilities to interact with her. In the last setting, the speaker (or rather: the writer) has the least control and no reliable information about his or her recipients. To account for this variation, we propose the use of the audience design model (Bell, 1984) (see §2.1).

In the paper, we first look at the use of discourse connectives along two dimensions of variation and show that there are systematic differences regarding the frequency of different forms of discourse connectives ( $\$ 4)$. Next, we show that the differences in the distribution of discourse connectives also reflect differences regarding the distribution 
of discourse relations in the different situational settings ( $\S 5)$. Finally, we discuss whether the different distribution of discourse relations in each setting reflects different strategies used to pursue a communicative purpose ( $(6)$, and how these might relate to audience design.

\section{Background}

\subsection{Discourse connectives in argumentative and persuasive text}

In this work, we focus on the use of explicitly marked discourse relations in persuasive text. In particular, we investigate how different dimensions of variation impact a speaker's linguistic behaviour during the production of argumentative texts. The approach we take is a comparative study of discourse connectives in persuasive texts produced by the same speaker, but in different discourse situations.

Our investigation takes the following two observations as its starting point. First, it has been shown that the use of discourse connectives and the distribution of explicit and implicit discourse relations is genre-dependent (see, e.g., Webber (2009) for written genres or Rehbein et al. (2016) for spoken texts). ${ }^{1}$ Second, Eckle-Kohler et al. (2015) show that certain discourse connectives are highly predictive features for distinguishing claims and premises in argumentative texts. ${ }^{2}$ This suggests that discourse connectives play a crucial role as strategic devices for persuasion. We follow O'Keefe (1990) and define persuasion as "a successful intentional effort at influencing another's mental state through communication in a circumstance in which the persuadee has some measure of freedom"(O'Keefe, 1990, p.5).

We distinguish persuasive from merely argumentative texts that rely on the neutral presentation of a complete set of claims and premises and weigh these against each other. In contrast, persuasive texts use additional rhetorical means to achieve their communicatve goal, such as rhetor-

\footnotetext{
${ }^{1}$ Due to space considerations, we refrain from including a discussion on the definition of register, genre and text type as there is a lack of agreement on the definition of those terms. In the paper, we try to avoid those terms and instead use the term discourse setting to refer to the different situational settings of text production.

${ }^{2}$ The authors, however, do not extend their study to discourse relations but, lacking DR annotations, only look at the word forms of discourse connectives. This is not optimal as most connectives are highly ambiguous and can express a number of different discourse relations.
}

ical questions, emotional and sentiment-loaded language, a high ratio of imagery, repetition, hyperbole, and more. In addition, presentational choices are made that select or focus on certain aspects of a topic or discourse entity, in order to validate the speaker's point of view and to support her communicative goals. This is often refered to as framing (Entman, 1993).

Stab and Gurevych (2014) look at argumentative texts and try to automatically identify claims and premises. They find that discourse connectives are often indicative of certain argument components. Tan et al. (2016) investigate persuasion strategies in online discussion forums. They also try to identify argument structure based on discourse connectives and report negative results for this approach, probably due to data sparseness. Felder (2015) shows how concessive and contrastive discourse connectives can be used to identify the central points of conflict in an argument, based on the selection or foregrounding of specific subtopics that are used to frame the discourse. A certain topic can, for example, be discussed against the background of moral or economic arguments, and thus appeal to different groups of people with differing political views (also see the work of Card et al. (2015, 2016) on media frames). In his work, Felder uses discourse connectives as signals for identifying conflicting framing strategies but does not investigate their strategic function in the discourse.

Kamalski et al. (2006) present two psycholinguistic experiments showing that the use of discourse connectives not only has a positive effect on the hearer's comprehension facility but also leads to a more positive evaluation of the text. This observation suggests that discourse connectives might be used as strategic devices in persuasive text.

\subsection{Audience design}

Work on accomodation (Giles et al., 1991) and audience design (Bell, 1984) has shown that language variation is not only influenced by social variables describing the speaker (e.g. age, gender, social class, etc.) but that speakers also adapt their style depending on who is listening. Bell investigated the speech of radio news broadcasters from different channels that targeted different audiences. He showed that the same broadcasters varied their linguistic style, depending on the 
channel. The audience design model has since been applied to many different discourse settings.

Litt (2012) extended the model for what he calls the "imagined audience", accounting for style shifts in situations where the real audience is not known to the speaker and thus the speaker adapts her style to a mental model of a hearer. This is relevant for many broadcasting media, for example for social media platforms such as forums, blogs or microtext messengers. Many studies have described and quantified effects of audience design in social media, looking at power relations, politeness and other variables of style shifts (Bramsen et al., 2011; Gilbert, 2012; Prabhakaran et al., 2012; niculescu mizil et al., 2012; DanescuNiculescu-Mizil et al., 2013; Pavalanathan and Eisenstein, 2015).

Applying the audience design model of Bell (1984) to our data, we have to account for different types of audiences. In the interview situation, the speaker is talking to one or more adressees who are known to the speaker and who are able to interact with her. In oral talks, the audience is visible to the speaker and can be directly addressed by her. However, the speaker usually has much less information about the hearers, and the audience has very limited means to actively take part in the communication even though there still is a certain amount of interaction through clapping, heckling or booing. This is different from the production situation of written articles where the text author has no knowledge or control about future readers of the text and thus the recipients can be considered as the "imagined audience", a mental model created in the mind of the author.

In the remainder of the paper, we present an annotation study where we apply the audience design model to our data to see how well it can explain the differences in the use of discourse connectives and explicit discourse relations as strategic devices for persuasion.

\section{Data}

The data we use in our annotation study are articles and talks by and interviews with Noam Chomsky. ${ }^{3}$ We created a corpus with 428,679 tokens of articles, 302,672 tokens of talks and 138,866 tokens of interviews. All data has been produced in a time period between 1985 and 2016.

\footnotetext{
${ }^{3}$ We collected the data from https://chomsky. info.
}

From the larger corpus, we selected a smaller sample for manual annotation with around 20,000 tokens per discourse setting. ${ }^{4}$ The smaller dataset was also controlled for topic. All articles in the sample focus on issues related to Gaza/Middle East/Palestine and the texts were selected from a smaller time range, covering the years from 2008 to 2014.

To take a first step towards investigating our hypothesis that discourse connectives are used as strategic devices for persuasion, we first explore how different forms of discourse connectives are used in persuasive texts by the same speaker, but produced in different situational settings.

In the next step, we investigate whether variation with regard to discourse connective form also reflects variation regarding the choice of discourse relations used to persue the communicative goal, or whether the observed variation can be explained simply by the use of different forms of discourse connectives that express the same discourse relation.

We argue that if we would find differences regarding the use of discourse relations in persuasive texts controlled for speaker and topic but produced in different situational settings, these differences could not be easily explained based on genre differences but would need a situational model that also accounts for the hearer/reader, such as the audience design model.

\section{Distribution of discourse connectives}

We start with an exploration of the distribution of discourse connectives in the three subcorpora. We follow the tradition of Biber's register analysis (Biber 1995) and perform a Principal Component Analysis (PCA), ${ }^{5}$ based on the frequency of 22 causal, concessive and contrastive discourse connective forms, to identify the main variables of variance. The set we use includes the following connectives: accordingly, although, because, but, conversely, hence, however, instead, nevertheless, nonetheless, nor, rather, since, so, still, thereby, therefore, though, thus, whereas, while, yet. We split the different texts in the large corpus in samples of 250 sentences each, count the frequency for each of the forms in the different samples and

\footnotetext{
${ }^{4}$ The token counts vary slightly as we did not cut off sentences but incuded all additional tokens until the end of the sentence.

${ }^{5}$ We use the FactoMineR library in the statistical software R (https: //www.r-project.org).
} 

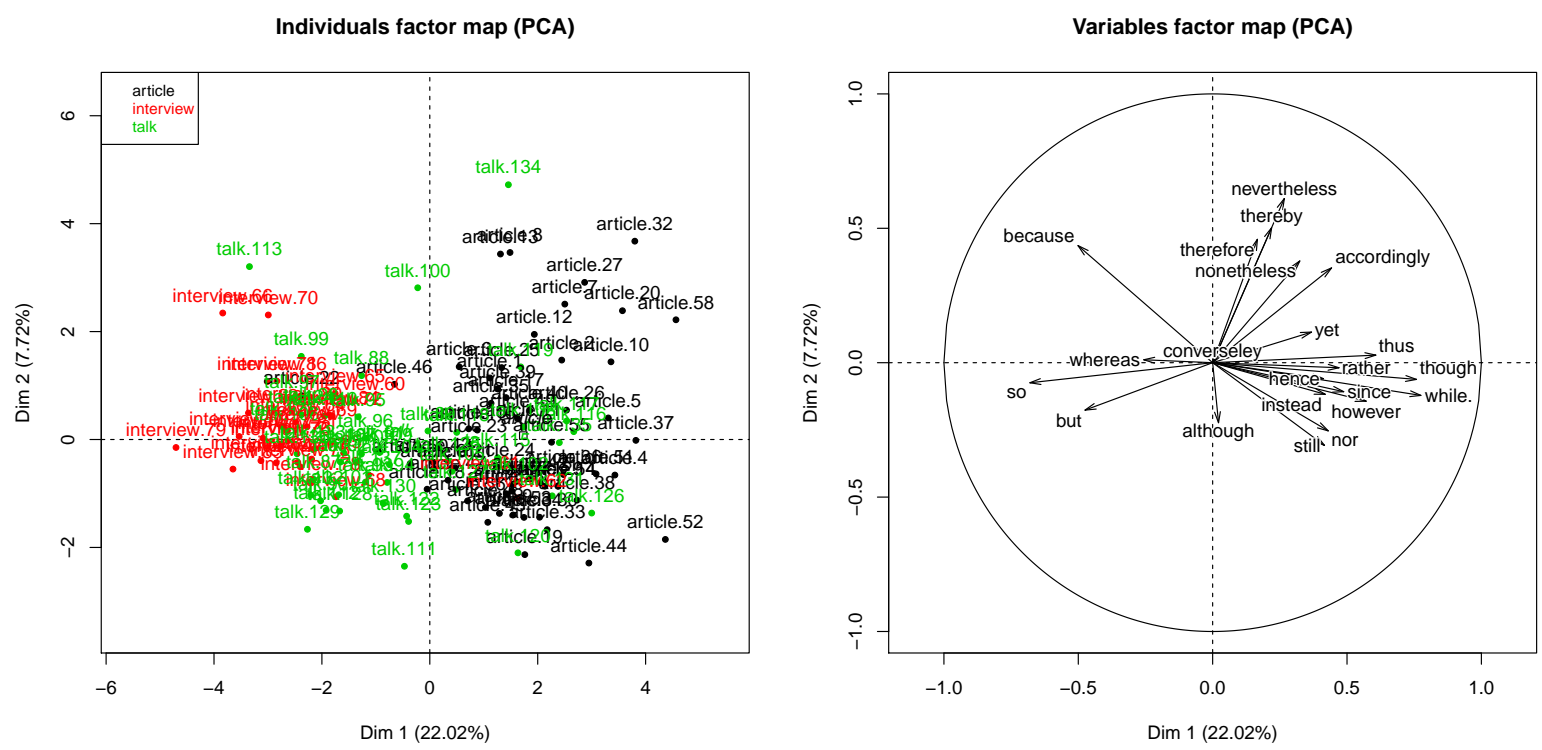

Figure 1: PCA on the larger dataset, showing the distribution of different discourse connective forms in persuasive articles, talks and interviews for the same speaker.

run the PCA on the data.

The PCA, despite having no other information but the frequency for each discourse connective, is able to separate the interviews from the articles along the first dimension. Figure 1 (left) shows how most of the interviews cluster at the left end of the dimension while the articles are positioned at the right end. The talks show a larger variance in the use of discourse connectives, and some talk segments show an overlap with the interviews while in other talks the use of discourse connectives is more similar to the one in the articles.

Figure 1 (right) identifies the discourse connectives most typical for written articles (though, while, thus, however) and those that are used in the less edited, dialogic interview data (because, so, but).

Our analysis clearly confirms what has been shown before (Webber, 2009; Rehbein et al., 2016), namely that the use of discourse connectives is highly genre-specific. In earlier genre studies, however, the different genres represented texts with different communicative functions, i.e. informative texts versus argumentative texts etc. Therefore, one might assume that the differences in the use of discourse connectives might reflect functional differences.

In our study, we try to eliminate this factor. We control for speaker and -in the smaller subcorpusalso for topic. All texts have a clear persuasive goal, i.e. to convince the audience from a particular political point of view. The main difference between the texts is the communication setting in which they were produced. This allows us to investigate the role that discourse connectives play for strategies of persuasion, tailored towards a particular setting and audience.

Given that the texts have the same persuasive function, we assume that the differences we observed reflect differences along two dimensions of variation that are correlated with the situational settings of text production. In a more detailed analysis with more features than just the counts for different connective forms, similar to Biber (1995); Biber and Conrad (2009); Passonneau et al. (2014), we would expect to find the two dimensions displayed in figure 2.

The first dimension distinguishes highly edited texts from less edited ones. Here the articles are positioned on the left end of the dimension, the talks can be located somewhere in the middle and the interviews as the least edited of the three text types are positioned at the right end. The second dimension concerns the interactional dimension of communication and sets monological texts apart from dialogical ones. Here, the articles can be placed at the monological end of the scale while the interviews are clearly dialogical and can thus be positioned at the other end of the dimension. The talks are mostly monological but allow for 


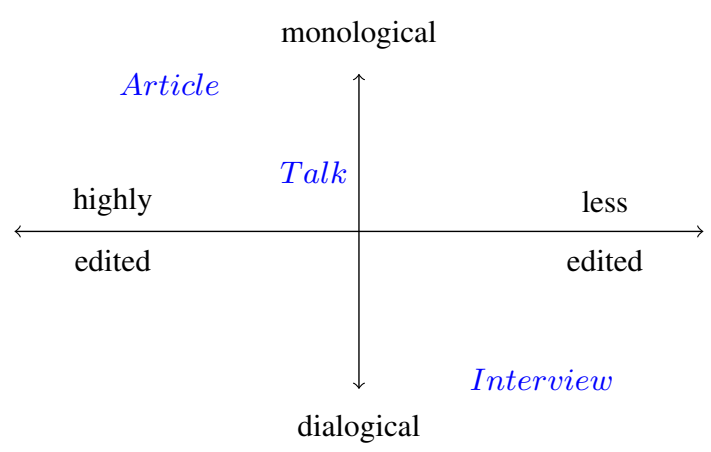

Figure 2: Dimensions of variation for different discourse settings.

some interaction with the audience.

These two dimensions of variation might explain the variance in the distribution of discourse connectives in the data, as the same discourse relations can be expressed via different connectives (or can also be left implicit). We thus hypothesize that the differences we observe will disappear when we look at the level of discourse relations instead of discourse connective forms. As there is no one-to-one correspondence between discourse connectives and discourse relations and automatic tools are not yet reliable enough, we need to manually disambiguate the relations in the data.

\section{Annotation study}

We present an annotation study where we annotate all instances of discourse connectives that can express a causal, contrastive or concessive discourse relation. We follow the framework of PDTB3 (Webber et al., 2016), a revised version of the Penn Discourse Treebank scheme (Prasad et al., 2008). The question we would like to answer is: Does the difference in distribution of discourse connectives in the texts shown above reflect differences in the distribution of discourse relations, or are the same relations expressed by different devices that are more adequate for a given discourse situation?

\subsection{Discourse relations in argumentative text}

The revised version of the PDTB comprises some changes to the relation hierarchy. Some relations in PDTB2 that were difficult to distinguish even for trained annotators have been merged in PDTB3, and inconsistencies regarding the annotation of the directionality of the relation have been removed. The direction describes the order of the arguments. In the PDTB scheme, Arg1 and Arg2

\begin{tabular}{|c|c|c|c|}
\hline LEVEL-1 & LEVEL-2 & LEVEL-1 & LEVEL-2 \\
\hline COMPARISON & $\begin{array}{l}\text { Contrast } \\
\text { Similarity } \\
\text { Concession }+/-\beta, \zeta\end{array}$ & TEMPORAL & $\begin{array}{l}\text { Synchronous } \\
\text { Asynchronous }\end{array}$ \\
\hline CONTINGENCY & $\begin{array}{l}\text { Cause }+/-\beta, \zeta \\
\text { Condition }+/-\beta, \zeta \\
\text { Neg. cond. }+/-\beta, \zeta \\
\text { Purpose }\end{array}$ & EXPANSION & $\begin{array}{l}\text { Conjunction } \\
\text { Disjunction } \\
\text { Equivalence } \\
\text { Instantiation } \\
\text { Level-of-detail } \\
\text { Substitution } \\
\text { Exception } \\
\text { Manner }\end{array}$ \\
\hline
\end{tabular}

Table 1: First two levels of hierarchy in the PDTB3 (level 3 encodes the direction of the relation, if applicable).

are determined by position in inter-sentential relations as well as in intra-sentential paratactic structures (e.g. coordinations). In intra-sentential subordinated structures, the subordinated clause is always labelled Arg2, regardless of its position. The new relation hierarchy is shown in table 1 .

Additionally, some relations can be marked as either pragmatic (epistemic) ( $\beta$; for implicit beliefs) or as speech acts $(\zeta) .^{6}$ These features should be understood as properties of the arguments, not of the relations (Webber et al., 2016). The examples below illustrate the different relation types relevant to our study. We follow the PDTB conventions and mark the first argument in italics and the second argument in boldface. The discourse connective that signals the relation is underlined.

- Concession: Although she was qualified, she didn't get the job.

- Contrast: Mary likes to read while John loves cooking.

- Cause: The street is wet because it rained last night.

- Cause + Belief $(+\beta)$ : She must be home because the light is on.

- Cause + Speechact $(+\zeta)$ : He's in Denver because he just called me an hour ago.

\subsection{Discourse connectives versus discourse relations}

We manually disambiguate all discourse connective forms in the smaller subcorpus that can express a causal, concessive or contrastive relation. The data includes 20,000 tokens from each discourse setting and was controlled for topic (table 2 ). The annotation was done by one trained linguist who had previous experience with PDTBstyle annotations. As annotation tool, we used

\footnotetext{
${ }^{6}$ For a distinction between, content, epistemic and speech act relations, see Sweetser (1990).
} 
the PDTB Annotator (Lee et al., 2016). We annotated all senses for instances of discourse connectives from a set of 22 word forms (see section 4). This resulted in 1,614 annotated instances (articles: 395, talks: 633, interviews: 586).

As there was only one annotator, we cannot report inter-annotator agreement for this task. However, in a comparable previous study on annotating PDTB-style discourse relations on English, the same annotator showed an IAA of $84.6 \%$ (percentage agreement) and a Fleiss' $\kappa$ of 0.797 (Rehbein et al., 2016).

Table 3 shows the distribution of causal, concessive and contrastive senses for the different discourse settings (see table 7 in the appendix for a complete list of all senses in PDTB3 (Webber et al., 2016)). We decided to normalise raw counts per sentence, as discourse relations mostly operate on the sentence or clause-level. ${ }^{7}$

The talks have the highest amount of signalled causal and concessive discourse relations in our corpus. The number of concessive relations, however, is smaller than in the article and interview subcorpora. In contrast to the use of discourse connective forms, the distribution of discourse relations found in the data cannot be explained with regard to the two dimensions of variation we discussed above (monologic-dialogic and less edited-highly edited).

However, when looking at more fine-grained sense distinctions, also taking the PDTB level three senses into account that encode the direction of the relation, we make an interesting observation (table 4). We can see that the higher number of causal relations in the talks reflects a more frequent use of RESULT-type relations while the distribution of REASON-type relations in the three discourse settings is fairly similar. If we look at concessive relations, we see that the higher frequency in the talks is caused only by Arg2-as-

${ }^{7}$ Results for a normalisation based on clauses were no
significantly different from a sentence-based normalisation.
\begin{tabular}{|l|r|r|r|r|}
\hline & token & sent & clause & annotations \\
\hline article & 20,020 & 822 & 2,062 & 395 \\
interview & 20,009 & 1,083 & 2,559 & 633 \\
talk & 20,011 & 1,123 & 2,623 & 586 \\
\hline total & 60,040 & 3,028 & 7,244 & 1,614 \\
\hline
\end{tabular}

Table 2: Subcorpus used in the annotation study (annotations lists the number of annotated relations for each discourse setting).

\begin{tabular}{|l|r|r|r|}
\hline LEVEL-2 & article & interview & talk \\
\hline \multicolumn{4}{|c|}{ raw counts } \\
\begin{tabular}{|l|r|r|r|}
\hline Cause & 41 & 73 & 101 \\
Contrast & 51 & 67 & 49 \\
Concession & 47 & 58 & 86 \\
\hline total & $\mathbf{1 3 9}$ & $\mathbf{1 9 8}$ & $\mathbf{2 3 6}$ \\
\hline \multicolumn{4}{|c|}{ normalised per sentence } \\
\hline Cause & 5.0 & 6.7 & $\mathbf{9 . 0}$ \\
Contrast & 6.2 & $\mathbf{6 . 2}$ & 4.4 \\
Concession & 5.7 & 5.3 & $\mathbf{7 . 7}$ \\
\hline total & $\mathbf{1 6 . 9}$ & $\mathbf{1 8 . 2}$ & $\mathbf{2 1 . 0}$ \\
\hline
\end{tabular}
\end{tabular}

Table 3: Distribution of causal, concessive and contrastive discourse relations (raw counts per discourse setting, and normalised counts per sentence)

\begin{tabular}{|l|l|r|r|r|}
\hline LEVEL-2 & LEVEL-3 & article & interv. & talk \\
\hline Cause & Reason & 2.43 & 2.12 & 2.49 \\
& Result & 2.55 & 4.43 & $\mathbf{6 . 5 0}$ \\
& Result $+\beta$ & 0.12 & 0.18 & 0.00 \\
\hline \multicolumn{5}{|c|}{$\beta$} \\
\hline \multirow{2}{*}{ Concession } & Arg1-denier & $\mathbf{0 . 8 5}$ & 0.55 & 0.18 \\
& Arg2-denier & 4.87 & 4.71 & $\mathbf{7 . 4 8}$ \\
\hline
\end{tabular}

Table 4: Amount of level-3 causal and concessive discourse relations (normalised per sentence; $\beta$ : belief)

denier relations while instances of Arg1-as-denier are more frequent in the article subcorpus. We also found one instance of Concession where we were not able to annotate the direction (see example below). As the example is from the interviews part of the corpus, we assume that it might be a performance error and exclude it from the analysis.

They can also be supplemented by various forms of direct action, such as what is now called " $B D S$," though that is only one of many tactical options.

To get a different perspective, we go back to the more coarse-grained level-2 senses but this time also include the SPEECHACT features $(\zeta$ ) (table 5). Here we can see that the crucial difference between the talks and the other two discourse settings regarding the CONCESSION relation can be traced back to the presence or absence of the $\zeta$ feature. SPEECHACT-type relations hardly ever occur in the articles and interviews but are quite frequent in the political talks, thus showing that it is not only difference between oral and written that triggers the use of speechact relations.

\begin{tabular}{|l|c|r|r|r|}
\hline LEVEL-2 & FEATURES & article & interview & talk \\
\hline \hline \multirow{2}{*}{ Concession } & $-\zeta$ & 5.2 & 4.9 & 5.6 \\
& $+\zeta$ & 0.5 & 0.4 & $\mathbf{2 . 0}$ \\
\hline
\end{tabular}

Table 5: Amount of concessive discourse relations with/without SPEECHACT (normalised per sentence; $\zeta$ : speech act) 


\section{Discussion}

So far, we have shown that persuasive texts produced by the same speaker in different situational settings do vary with regard to the distribution of discourse connective forms in the texts, and that this might reflect differences along the dimension of editedness and the degree of interaction in the texts.

Next, we have shown that the variation with regard to discourse connective forms in persuasive texts controlled for topic and speaker but produced in different discourse settings is not only due to stilistic choices made by the speaker. Instead, we also found variation on the level of discourse relations, with causal result and concessive speechact relations being used far more often in the talks than in the other two discourse settings.

This variation cannot be easily explained with regard to genre differences as looking at the distribution of discourse relations should abstract away the stilistic differences between different connective forms (e.g. formal-informal, highly editedless edited). As we assume that that function of the texts is the same (persuasive text), we hypothesize that the variation in the distribution of discourse relations in each communicative setting reflects the use of different persuasive strategies employed to persue the communicative goal.

\subsection{Causal relations}

Let us start by looking at the causal relations. While the frequency of REASON relations was more or less equal in all three subcorpora, we observed a substantially higher amount of RESULT relations in the talks. Examples (2), (3) show typical examples for REASON and RESULT relations in the talk subcorpus.

He was removed from office soon af-
ter because he was considered too soft-
hearted.

For US leaders, aggression means resistance. $\underline{\text { So, }}$ anyone who resists the United States is guilty of aggression. RESULT

The first example describes an event in the real world (removed from office) and presents the cause for this event (being too soft-harted). It can thus be categorised as a consequence-cause relation. Example (3), however, does not describe an event but rather presents two claims, with the first claim providing the pragmatic justification for the second one. Please note that instances like (3) are not annotated as implicit beliefs. The reason for this becomes clear when modifying example (3), as shown below:

For US leaders, aggression means resistance. So I believe, anyone who resists the United States is guilty of aggression.

The meaning of (4) is different from (3) where the subject of consciousness holding the belief expressed in Arg2 are the US leaders while in the modified example the subject of consciousness is the speaker. ${ }^{8}$

Looking at more examples, one striking feature is the frequent use of the first person plural pronoun (we; examples (5)-(7) below) in the talks.

(5) The countries we wanted to sell it to did n't have dollars so we had to provide them with dollars.

RESULT

And that makes sense if we own the world $\underline{\text { so }}$ any active resistance is aggression against us.

RESULT

We did it so therefore it's efforts to do good.

RESULT

To check whether first person pronouns in general are more frequent in the talk subcorpus, we counted the number of occurrences of $I$ and we for each discourse setting. Table 6 shows that, as expected, the written texts have the lowest number of first person pronouns. First person pronouns are considered a marker of involvement and are often used to discriminate spoken from written registers (Biber, 1995, p.225). Here, however, they not only distinguish the written from the spoken texts but also set apart the talks from the interviews. While in the latter both $I$ and we are used with high frequency, in the talks we observe a significantly higher number of first person plural usage.

\subsection{Concession and SpeechAct}

Another difference between the three discourse settings concerns the substantially higher number

\footnotetext{
${ }^{8}$ The two claims in (3) are presented as facts in the world with the second one following from the first, and not as epistemic beliefs. We therefore annotate them as content relations in the sense of Sweetser (1990).
} 


\begin{tabular}{|l|r|r|r|}
\hline 1.ps.pron & article & interview & talk \\
\hline we & 43 & 86 & 143 \\
$I$ & 13 & 79 & 49 \\
\hline all tokens & 20,020 & 20,009 & 20,011 \\
\hline
\end{tabular}

Table 6: Frequency of 1 . person pronouns $I$, we in the three discourse settings.

of SPEECHACT relations in the talks. This can not be ascribed to the medium, i.e. spoken language, as this relation type is not only rare in the written articles but also hardly ever occurs in the interviews. Below are typical examples for CONCESSION + SPEECHACT in the corpus.

I hope I don't have to describe it to you, but it killed several million people, destroyed three countries.

I won't go through the rest of the history but it continues pretty much like that.

I won't run through the whole story but the basic facts are clear.

Again, we observe a high number of first person pronouns, here mostly the singular form $I$. All of these examples anchor the speech act in the hereand-now by referring to the discourse situation and also by referencing a common ground shared between speaker and audience. By doing so, the speaker presumes that the hearers agree with his point of view even without giving further details on the topic under discussion (examples (8)-(10)).

This communicative strategy requires that the Ground (Langacker, 1990), i.e. the immediate circumstances of the speech event such as time and space, are shared between speaker and audience. This explains why this strategy cannot be used in written articles where the speaker only has access to an imagined audience at best, and no interaction is possible. In the interview setting, however, the increased level of interaction might interfere with the speaker's construction of the shared common ground.

\subsection{Political talks as oral narratives}

Based on the results of our annotation study, we hypothesize that the differences in the distribution of discourse relations reflect the use of different strategies of persuasion in the different communicative settings. We suggest that it might make sense to consider persuasive political talks as oral narratives in the sense of Labov and Waletzky
(1967), with the function of "transfer[ing] experience from one person to another through oral narratives of personal experience" (Labov, 2010). This fits well with the high number of personal pronouns in the talks which not only reflect a high amount of involvement but also give credibility to the narrative. Furthermore, the use of first person pronouns helps to create an impression of intimacy between speaker and audience and also lends the narrative authenticity (Malti-Douglas, 1988, p.93).

These ideas, however, are highly speculative and need to be tested empirically on a larger basis and including more than one speaker.

\section{Conclusions}

We presented an annotation study where we investigated the use of discourse connectives and disourse relations in persuasive texts. The texts in our corpus are controlled for speaker and topic but produced in different communicative settings. We observed a substantial variation in the use of discourse connective forms and relations. While the first can be easily explained by genre differences, we argue that the second variation concerning the use of discourse relations might reflect different strategies of persuasion, and that models of audience design might prove useful for understanding this variation.

\section{Acknowledgments}

I would like to thank the reviewers for their helpful comments. This research has been conducted within the Leibniz Science Campus "Empirical Linguistics and Computational Modeling", funded by the Leibniz Association under grant no. SAS2015-IDS-LWC and by the Ministry of Science, Research, and Art (MWK) of the state of BadenWürttemberg.

\section{References}

Allan Bell. 1984. Language style as audience design. Language in society, 13(2):145-204.

Doublas Biber and Susan Conrad. 2009. Register, genre, and style. Cambridge: Cambridge University Press.

Douglas Biber. 1995. Variation across speech and writing. Publisher: Cambridge University Press.

Philip Bramsen, Martha Escobar-Molano, Ami Patel, and Rafael Alonso. 2011. Extracting social power 
relationships from natural language. In The 49th Annual Meeting of the Association for Computational Linguistics: Human Language Technologies, HLT 2011, pages 773-782.

Dallas Card, Amber E. Boydstun, Justin H. Gross, Philip Resnik, and Noah A. Smith. 2015. The media frames corpus: Annotations of frames across issues. In Proceedings of the 53rd Annual Meeting of the Association for Computational Linguistics and the 7th International Joint Conference on Natural Language Processing of the Asian Federation of Natural Language Processing, ACL 2015, pages 438-444, Beijing, China.

Dallas Card, Justin H. Gross, Amber E. Boydstun, and Noah A. Smith. 2016. Analyzing framing through the casts of characters in the news. In Proceedings of the 2016 Conference on Empirical Methods in Natural Language Processing, EMNLP 2016, pages 1410-1420, Austin, Texas, USA.

Cristian Danescu-Niculescu-Mizil, Moritz Sudhof, Dan Jurafsky, Jure Leskovec, and Christopher Potts. 2013. A computational approach to politeness with application to social factors. In The 51st Annual Meeting of the Association for Computational Linguistics, ACL 2013, pages 250-259.

Judith Eckle-Kohler, Roland Kluge, and Iryna Gurevych. 2015. On the role of discourse markers for discriminating claims and premises in argumentative discourse. In Proceedings of the 2015 Conference on Empirical Methods in Natural Language Processing, EMNLP 2015, Lisbon, Portugal, September 17-21, 2015, pages 2236-2242.

Robert M. Entman. 1993. Framing: Toward clarification of a fractured paradigm. Communication, 43(4):51-58.

Ekkehard Felder. 2015. Lexik und grammatik der agonalität in der linguistischen diskursanalyse. In Heidrun Kmper and Ingo Warnke, editors, Diskurs - interdisziplinär. Zugänge, Gegenstände, Perspektiven, pages 87-121. Berlin/Boston: de Gruyter.

Eric Gilbert. 2012. Phrases that signal workplace hierarchy. In Proceedings of the ACM 2012 Conference on Computer Supported Cooperative Work, CSCW '12, pages 1037-1046.

Howard Giles, Justine Coupland, and Nikolas Coupland. 1991. Contexts of Accommodation: Developments in Applied Sociolinguistics. Cambridge: Cambridge University Press.

Judith Kamalski, Leo Lentz, and Ted Sanders. 2006. Effects of coherence marking on the comprehension and appraisal of discourse. In Cognitive Science Conference.

William Labov. 2010. Oral narratives of personal experience. In Cambridge Encyclopedia of the Language Sciences. Cambridge: Cambridge University Press.
William Labov and Joshua Waletzky. 1967. Narrative analysis: oral versions of personal experience. In Essays on the Verbal and Visual Arts: Proceedings of the 1966 Annual Spring Meeting of the American Ethnological Society, pages 12-44. University of Washington Press, Seattle.

Ronald Langacker. 1990. Subjectification. Cognitive Linguistics, 1:5-38.

Alan Lee, Rashmi Prasad, Bonnie L. Webber, and Aravind K. Joshi. 2016. Annotating discourse relations with the PDTB annotator. In The 26th International Conference on Computational Linguistics, Proceedings of the Conference System Demonstrations, COLING 2016, pages 121-125.

Eden Litt. 2012. Knock, knock. who's there? the imagined audience. Journal of Broadcasting \& Electronic Media, 56:330-345.

Fedwa Malti-Douglas. 1988. Blindness and Autobiography: Al-Ayyam of Taha Husayn. Princeton University Press.

Cristian Danescu niculescu mizil, Lillian Lee, Bo Pang, and Jon Kleinberg. 2012. Echoes of power: Language effects and power differences in social interaction. In The 21st International Conference on World Wide Web, WWW 2012, pages 699-708.

Daniel J. O'Keefe. 1990. Persuasion: Theory and research. Newbury Park, CA: Sage.

Rebecca J. Passonneau, Nancy Ide, Songqiao Su, and Jesse Stuart. 2014. Biber redux: Reconsidering dimensions of variation in american english. In The 25th International Conference on Computational Linguistics, COLING 2014, pages 565-576.

Umashanthi Pavalanathan and Jacob Eisenstein. 2015. Audience-modulated variation in online social media. American Speech, 90(2):187-213.

Vinodkumar Prabhakaran, Owen Rambow, and Mona Diab. 2012. Predicting overt display of power in written dialogs. In The 2012 Conference of the North American Chapter of the Association for Computational Linguistics: Human Language Technologies, NAACL 2012, pages 518-522.

R. Prasad, N. Dinesh, A. Lee, E. Miltsakaki, L. Robaldo, A.K. Joshi, and B.L. Webber. 2008. The penn discourse treebank 2.0. In Proceedings of the 6 International Conference on Language Resources and Evaluation, LREC'08. European Language Resources Association.

Ines Rehbein, Merel Scholman, and Vera Demberg. 2016. Annotating discourse relations in spoken language: A comparison of the PDTB and CCR frameworks. In Proceedings of the Tenth International Conference on Language Resources and Evaluation LREC 2016, Portorož, Slovenia, May 23-28, 2016. 
Christian Stab and Iryna Gurevych. 2014. Identifying argumentative discourse structures in persuasive essays. In Conference on Empirical Methods in Natural Language Processing, EMNLP 2014, pages 4656.

Eve Sweetser. 1990. From Etymology to Pragmatics. Cambridge University Press, Cambridge.

Chenhao Tan, Vlad Niculae, Cristian DanescuNiculescu-Mizil, and Lillian Lee. 2016. Winning arguments: Interaction dynamics and persuasion strategies in good-faith online discussions. In The 25th International Conference on World Wide Web, WWW 2016, pages 613-624.

Bonnie L. Webber. 2009. Genre distinctions for discourse in the penn treebank. In ACL 2009, Proceedings of the 47th Annual Meeting of the Association for Computational Linguistics and the 4th International Joint Conference on Natural Language Processing of the AFNLP, 2-7 August 2009, Singapore, pages 674-682.

Bonnie L. Webber, Rashmi Prasad, Alan Lee, and Aravind K. Joshi. 2016. A discourse-annotated corpus of conjoined vps. In The 10th Linguistic Annotation Workshop, LAW 2016.

\section{A Appendices}




\begin{tabular}{|c|c|c|}
\hline LEVEL-1 & LEVEL-2 & LEVEL-3 \\
\hline \multirow[b]{2}{*}{ TEMPORAL } & SYNCHRONOUS & - \\
\hline & ASYNCHRONOUS & $\begin{array}{l}\text { Precedence } \\
\text { SuCCESSION }\end{array}$ \\
\hline COMPARISON & $\begin{array}{l}\text { CONTRAST } \\
\text { SIMILARITY } \\
\text { CONCESSION }+/-\beta, \zeta\end{array}$ & $\begin{array}{l}- \\
- \\
\text { Arg1-as-denier } \\
\text { Arg1-as-denier }\end{array}$ \\
\hline \multirow{4}{*}{ CONTINGENCY } & Cause $+/-\beta, \zeta$ & $\begin{array}{l}\text { Reason } \\
\text { Result }\end{array}$ \\
\hline & CONDITION $+/-\zeta$ & $\begin{array}{l}\text { Arg1-as-cond } \\
\text { Arg2-as-cond }\end{array}$ \\
\hline & NEGATIVE CONDITION $+/-\zeta$ & $\begin{array}{l}\text { Arg1-as-negcond } \\
\text { Arg2-as-negcond }\end{array}$ \\
\hline & PURPOSE & $\begin{array}{l}\text { Arg1-as-goal } \\
\text { Arg1-as-goal }\end{array}$ \\
\hline \multirow{8}{*}{ EXPANSION } & CONJUNCTION & - \\
\hline & DISJUNCTION & - \\
\hline & EQUIVALENCE & - \\
\hline & INSTANTIATION & - \\
\hline & LEVEL-OF-DETAIL & $\begin{array}{l}\text { Arg1-as-detail } \\
\text { Arg2-as-detail }\end{array}$ \\
\hline & Substitution & $\begin{array}{l}\text { Arg1-as-subst } \\
\text { Arg2-as-subst }\end{array}$ \\
\hline & EXCEPTION & $\begin{array}{l}\text { Arg1-as-excpt } \\
\text { Arg2-as-excpt }\end{array}$ \\
\hline & MANNER & $\begin{array}{l}\text { Arg1-as-manner } \\
\text { Arg2-as-manner }\end{array}$ \\
\hline
\end{tabular}

Table 7: Sense hierarchy in the PDTB3 (level 3 encodes the direction of the relation, if applicable). 